\title{
DISCOVERY AND CHARACTERIZATION OF A FAINT STELLAR COMPANION TO THE
} A3V STAR $\zeta$ VIRGINIS

This article has been downloaded from IOPscience. Please scroll down to see the full text article.

2010 ApJ 712421

(http://iopscience.iop.org/0004-637X/712/1/421)

The Table of Contents and more related content is available

Download details:

IP Address: 131.215.193.213

The article was downloaded on 26/03/2010 at 21:29

Please note that terms and conditions apply. 


\title{
DISCOVERY AND CHARACTERIZATION OF A FAINT STELLAR COMPANION TO THE A3V STAR $\zeta$ VIRGINIS
}

\author{
Sasha Hinkley ${ }^{1,13}$, Ben R. Oppenheimer ${ }^{2}$, Douglas Brenner ${ }^{2}$, Neil Zimmerman ${ }^{2,3}$, Lewis C. Roberts Jr. ${ }^{4}$, Ian R. Parry ${ }^{5}$, \\ Rémi Soummer $^{6}$, Anand Sivaramakrishnan ${ }^{2,7,8}$, Michal Simon ${ }^{8}$, Marshall D. Perrin $^{9,14}$, David L. King ${ }^{5}$, James \\ P. Lloyd ${ }^{10}$, Antonin Bouchez $^{11}$, Jennifer E. Roberts ${ }^{4}$, Richard Dekany $^{11}$, Charles Beichman ${ }^{12}$, Lynne Hillenbrand $^{1}$, \\ Rick Burruss ${ }^{4}$, Michael Shao ${ }^{4}$, and Gautam Vasisht ${ }^{4}$ \\ ${ }^{1}$ Department of Astronomy, California Institute of Technology, 1200 East California Boulevard, MC 249-17, Pasadena, CA 91125, USA \\ ${ }^{2}$ Astrophysics Department, American Museum of Natural History, Central Park West at 79th Street, New York, NY 10024, USA \\ ${ }^{3}$ Department of Astronomy, Columbia University, 550 West 120th Street, New York, NY 10027, USA \\ ${ }^{4}$ Jet Propulsion Laboratory, California Institute of Technology, 4800 Oak Grove Dr., Pasadena, CA 91109, USA \\ ${ }^{5}$ Institute of Astronomy, University of Cambridge, Madingley Road, Cambridge CB3 OHA, UK \\ ${ }^{6}$ Space Telescope Science Institute, 3700 San Martin Drive, Baltimore, MD 21218, USA \\ ${ }^{7}$ NSF Center for Adaptive Optics, CA, USA \\ ${ }^{8}$ Stony Brook University, NY, USA \\ ${ }^{9}$ UCLA Department of Astronomy, CA, USA \\ ${ }^{10}$ Department of Astronomy, Cornell University, Ithaca, NY 14853, USA \\ ${ }^{11}$ Caltech Optical Observatories, California Institute of Technology, Pasadena, CA 91125, USA \\ 12 NASA Exoplanet Science Institute, California Institute of Technology, Pasadena, CA 91125, USA \\ Received 2009 November 2; accepted 2010 February 5; published 2010 March 2
}

\begin{abstract}
Through the combination of high-order adaptive optics and coronagraphy, we report the discovery of a faint stellar companion to the A3V star $\zeta$ Virginis. This companion is $\sim 7$ mag fainter than its host star in the $H$ band, and infrared imaging spanning 4.75 years over five epochs indicates this companion has common proper motion with its host star. Using evolutionary models, we estimate its mass to be $0.168_{-0.016}^{+0.012} M_{\odot}$, giving a mass ratio for this system $q=0.082_{-0.008}^{+0.007}$. Assuming the two objects are coeval, this mass suggests an M4V-M7V spectral type for the companion, which is confirmed through integral field spectroscopic measurements. We see clear evidence for orbital motion from this companion and are able to constrain the semimajor axis to be $\gtrsim 24.9$ AU, the period $\gtrsim 124 \mathrm{yr}$, and eccentricity $\gtrsim 0.16$. Multiplicity studies of higher mass stars are relatively rare, and binary companions such as this one at the extreme low end of the mass ratio distribution are useful additions to surveys incomplete at such a low mass ratio. Moreover, the frequency of binary companions can help to discriminate between binary formation scenarios that predict an abundance of low-mass companions forming from the early fragmentation of a massive circumstellar disk. A system such as this may provide insight into the anomalous X-ray emission from A stars, hypothesized to be from unseen late-type stellar companions. Indeed, we calculate that the presence of this M-dwarf companion easily accounts for the X-ray emission from this star detected by ROSAT.
\end{abstract}

Key words: instrumentation: adaptive optics - methods: data analysis - stars: individual (HIP66249, HR5107) techniques: image processing

Online-only material: color figures

\section{INTRODUCTION}

Stars with spectral type A have long shown evidence for surprising circumstellar disk structures (Smith \& Terrile 1984; Kalas et al. 2005; Golimowski et al. 2006; Oppenheimer et al. 2008) and stars with spectral type $\sim \mathrm{A} 6$ and earlier have become increasingly targeted for low-mass companions through high-contrast imaging (Hinz et al. 2006; Hinkley et al. 2007; Heinze et al. 2008; Oppenheimer \& Hinkley 2009) resulting in detections of several low-mass companions (Kalas et al. 2008; Marois et al. 2008). Indeed, Johnson et al. (2007) suggest that the frequency of planet occurrence around A-type stars is twice that of solar-mass stars. In addition to planetarymass companions, the frequency and mass ratio distributions of stellar-mass companions to nearby A stars can help constrain binary formation scenarios-such as models based on the more massive primary star dynamically capturing a lower mass companion (McDonald \& Clarke 1993), or a picture relying on

\footnotetext{
${ }^{13}$ Sagan Fellow.

${ }^{14}$ NSF Postdoctoral Fellow.
}

initial fragmentation within a protostellar cloud, e.g., Burkert \& Bodenheimer (1996). Although some multiplicity studies of A and B stars have been conducted-e.g., the Shatsky \& Tokovinin (2002) and Kouwenhoven et al. (2005) surveys of Sco OB2-a comprehensive statistical picture of multiplicity around these massive stars, based on both cluster and field objects, has yet to emerge. Observations of massive, early-type stars may serve as important boundary-type systems, to which models of formation must conform. Specifically, an abundance of brown dwarf/ M-dwarf companions to A stars would lend support to recent models describing the formation of these objects through the fragmentation of an initially massive circumstellar disk (Kratter et al. 2010; Stamatellos \& Whitworth 2009).

Moreover, the frequency of stellar companions to A stars may be related to their anomalous source of X-rays. Since A stars have shallow or non-existent convective regions in their envelopes, they lack a significant dynamo effect, and can be expected to display negligible X-ray emission. Meanwhile, M dwarfs are well-known sources of X-rays (Fleming et al. 1993). Schmitt et al. (1985) suggested that unseen late-type stellar companions to A stars may be the source of the X-rays. Indeed, 
Table 1

Fundamental Parameters for $\zeta$ Virginis A

\begin{tabular}{lc}
\hline \hline \multicolumn{1}{c}{ Parameter } & Value \\
\hline Identifiers & HIP66249, HR5107, \\
& HD118098 \\
Spectral type (1) & A3V \\
$V$ magnitude (2) & 3.40 \\
Parallax (mas) (3) & $44.55 \pm 0.90$ \\
R.A., decl. proper motion $\left(\right.$ mas yr $\left.^{-1}\right)(3)$ & $-278.89 \pm 0.83,48.56 \pm 0.71$ \\
Radial velocity $\left(\mathrm{km} \mathrm{s}^{-1}\right)(4)$ & $-13.2 \pm 0.3$ \\
\hline
\end{tabular}

References. (1) Gray et al. 2003; (2) Hoffleit \& Jaschek 1982; (3) Perryman et al. 1997; (4) Abt \& Biggs 1972; Lagrange et al. 2009.

Schröder \& Schmitt (2007) find that the majority of nearby X-ray emitting A stars have some evidence of possessing lowmass companions, likely responsible for the X-ray emission. Moreover, Zimmerman et al. (2010) have recently discovered a mid-M dwarf companion to the nearby A-star Alcor, a ROSAT source.

\subsection{Previous Studies of $\zeta$ Virginis}

The star $\zeta$ ("Zeta") Virginis (HIP66249, A3V, $V=3.40$-see Table 1, hereafter $\zeta$ Vir), is a target in our on-going high-contrast imaging program (Oppenheimer et al. 2004; Sivaramakrishnan et al. 2007; Hinkley et al. 2008). This nearby (22.45 pc) star has been previously used as a calibrator star for interferometric work (Akeson et al. 2009), and has also been followed with the HARPS survey for radial velocity variations (Lagrange et al. 2009). No such variations were found. The Bright Star Catalogue (Hoffleit \& Jaschek 1982) lists $\zeta$ Vir as a member of the Hyades moving group. There is some spread in the derived age of this group. Although some authors claim ages as high as $625 \mathrm{Myr}$ (Perryman et al. 1998) or $650 \mathrm{Myr}$ (Lebreton et al. 2001), Rieke et al. (2005) cite the age of this star as $505 \mathrm{Myr}$. Rather than trying to establish the membership of this star in the Hyades moving group, and then adopt the moving group age for the star, we simply adopt the 505 Myr age of Rieke et al. (2005) for the analysis in this paper. $\zeta$ Vir has indeed been observed by ROSAT (Huensch et al. 1998), and is listed as a single star, with an X-ray brightness $\left(L_{x}=1.07 \times 10^{28} \mathrm{erg} \mathrm{s}^{-1}\right)$. Despite the fact that there is a $20^{\prime \prime}$ offset between X-ray and optical positions, the ROSAT catalog lists a $14^{\prime \prime}$ uncertainty on the position of $\zeta$ Vir. Such a $1.5 \sigma$ positional offset easily leaves open the possibility that the observed X-ray source is in fact located at $\zeta$ Vir. The $\zeta$ Vir system has a radial velocity of $-13.2 \mathrm{~km} \mathrm{~s}^{-1}$ as mentioned in Abt \& Biggs (1972) and Kilkenny et al. (1998) found this radial velocity to be constant at the $1-2 \mathrm{~km} \mathrm{~s}^{-1}$ level, i.e., lacking a companion, using it as one of their standard calibrator stars.

Speckle observations at the Canada-France-Hawaii Telescope in the optical (McAlister et al. 1993) did not find a binary companion for this star; however, this is not surprising given the comparatively low dynamic range ( $\Delta M \sim 3 \mathrm{mag}$ ) of this technique. Patience et al. (2001) specifically carried out a survey of bright early-type stars both in the field and in clusters to search for companions using adaptive optics (AO), but no mention of this star is listed.

\section{OBSERVATIONS}

We have imaged the $\zeta$ Vir system using two observing programs, each with different instruments: a coronagraph working together with an infrared camera, and a newly commissioned

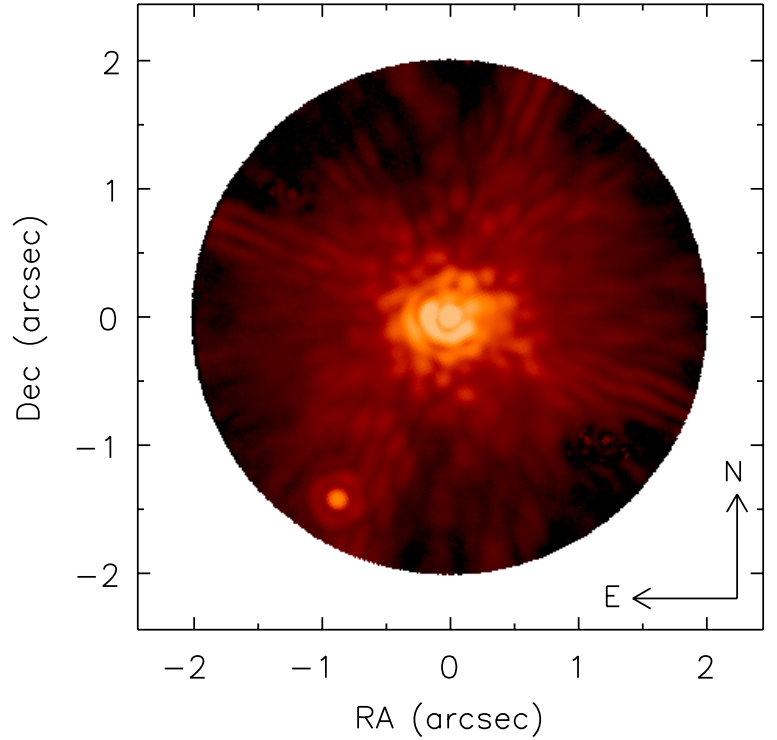

Figure 1. $60 \mathrm{~s} H$-band $(1.65 \mu \mathrm{m})$ image of the star $\zeta$ Vir taken on 2004 June 12 (UT) at the AEOS telescope. In this discovery image, the AO system is on, and the star has been coronagraphically occulted behind our 455 mas occulting mask. A faint stellar companion, $\zeta$ Vir B, 7 mag fainter than the host star and sharing common proper motion with the host star, is apparent at the bottom left of the image.

(A color version of this figure is available in the online journal.)

coronagraph which employs an integral field spectrograph (IFS) as the primary science camera. We describe each observing program below.

\section{1. "The Lyot Project," a Coronagraphic Imager at AEOS}

The first instrument, "The Lyot Project" (Oppenheimer et al. 2004; Sivaramakrishnan et al. 2007), was a diffraction-limited classical Lyot coronagraph (Lyot 1939; Sivaramakrishnan et al. 2001) working with the AO system on the $3.63 \mathrm{~m} \mathrm{AEOS}$ telescope on Haleakala, Hawaii (Roberts \& Neyman 2002). Our images were gathered using "Kermit," an infrared camera (Perrin et al. 2003), with a 13.5 mas pixel $^{-1}$ plate scale, and differential polarimetry mode for detection of diffuse circumstellar material (Hinkley et al. 2009). Images of $\zeta$ Vir in $J, H$, and $K$ bands were obtained using this instrument over three epochs spanning three years as listed in Table 2. Our coronagraph used a focal plane mask with a 455 mas diameter (4.9 $\lambda / D$ at $H$ band), as well as its own internal tip/tilt system. Images in the $J, H$, and $K$ bands were obtained during the second and third epochs, while only $H$ band was obtained on the first. To calibrate our photometry, we also obtained $1 \mathrm{~s}$ unocculted images in addition to the coronagraphically occulted images. In this setup, the target is more than $1^{\prime \prime}$ away from our occulting mask. The raw data images, both occulted and unocculted, required a mix of both traditional data reduction steps (e.g., dark current subtraction, bad-pixel masking, flat-field correction) as well as some techniques customized for the infrared camera. More details on the data reduction are given in Soummer et al. (2006) and Digby et al. (2006).

\section{2. "Project 1640," a Coronagraphic Integral Field Spectrograph at Palomar}

The second instrument used to image the $\zeta$ Vir system is a new instrument (Hinkley et al. 2008) recently commissioned on the 200-in Hale Telescope at Palomar Observatory. This 
Table 2

Observations and Astrometric Distance Between Primary and Companion for the $\zeta$ Vir System

\begin{tabular}{lccccr}
\hline \hline Epoch & MJD & Wavelength & Observing Method & Separation (mas) & PA (Degrees East of North) \\
\hline 1 & 53168.2839 & $H$ band & Lyot coronagraph & $1846 \pm 9$ & $144.7 \pm 0.1$ \\
2 & 53507.3742 & $J, H, K$ bands & Lyot coronagraph & $1830 \pm 3$ & $146 \pm 1.0$ \\
3 & 54257.5378 & $J, H, K$ bands & Lyot coronagraph & $1814 \pm 16$ & $147.4 \pm 0.1$ \\
4 & 54657.1548 & $1.1-1.8 \mu$ m (IFS) & APLC + IFS & $1790 \pm 12$ & $149.8 \pm 0.1$ \\
5 & 54904.4445 & $1.1-1.8 \mu$ m (IFS) & APLC + IFS & $1779 \pm 12$ & $151.0 \pm 0.2$ \\
\hline
\end{tabular}

instrument, termed "Project 1640," is a coronagraph integrated with an IFS spanning the $J$ and $H$ bands $(1.05-1.75 \mu \mathrm{m})$. The IFS+Coronagraph package is mounted on the Palomar AO system (Dekany et al. 1998), which in turn is mounted at the Cassegrain focus of the Hale Telescope. The coronagraph is an Apodized-Pupil Lyot coronagraph (APLC; Soummer 2005), an improvement of the classical Lyot coronagraph (Sivaramakrishnan et al. 2001). This coronagraph uses a 370 mas diameter (5.37 $\lambda / D$ at $H$ band) focal plane mask. The IFS, or hyper-spectral imager, is a microlens-based spectrograph which can simultaneously obtain $4 \times 10^{4}$ spectra across our $4^{\prime \prime} \times 4^{\prime \prime}$ field of view. Each microlens subtends 19.2 mas on the sky and a dispersing prism provides a spectral resolution $(\lambda / \Delta \lambda) \sim 32$.

The IFS focal plane consists of $4 \times 10^{4}$ spectra that are formed by dispersing the pupil images created by each microlens. To build a data cube, the data pipeline uses a library of images made in the laboratory with a tunable laser, which spans the operating wavelength band of the instrument. Each image contains the response of the IFS to laser emission at a specific wavelengtha matrix of point-spread functions (PSFs). Each laser reference image is effectively a key showing what regions of the $4 \times 10^{4}$ spectra landing on the detector correspond to a given central wavelength. Each laser reference image is cross correlated with the focal plane spectra to extract each wavelength channel.

The pixel scales for each instrument were calculated by imaging four binary stars (HIP107354, HIP171, HIP88745, and WDS11182+3132) with high-quality orbits with small astrometric residuals (Hartkopf et al. 2001). The pixel scale is calculated by performing a least-squares fit between these predicted separations and the pixel separation in our data.

\section{THE COMPANION}

Here we report the discovery of a faint stellar companion to $\zeta$ Vir, hereafter $\zeta$ Vir B. The discovery image is shown in Figure 1. To our knowledge, the existence of this companion has not been reported previously.

\subsection{Common Proper Motion Analysis}

The astrometric measurements for the primary/companion separation are presented in Table 2. For the first and third epochs, the astrometric positions of the two stars were obtained from images in which the primary star was occulted. In these cases, a centroid to each PSF was calculated as part of a best-fit radial profile measurement. With coronagraphic imaging, the exact position of the occulted star is difficult to determine. The uncertainty can be estimated using binary stars, in which one of the binary members is occulted (Digby et al. 2006). For all but the first epoch, we used a physical mask with a superimposed grid (Sivaramakrishnan \& Oppenheimer 2006), which produces fiducial reference spots indicating the position of the host star to within $\sim 10$ mas. The second, fourth, and fifth epochs contained fully unocculted data with sufficiently high signal-to-noise ratio

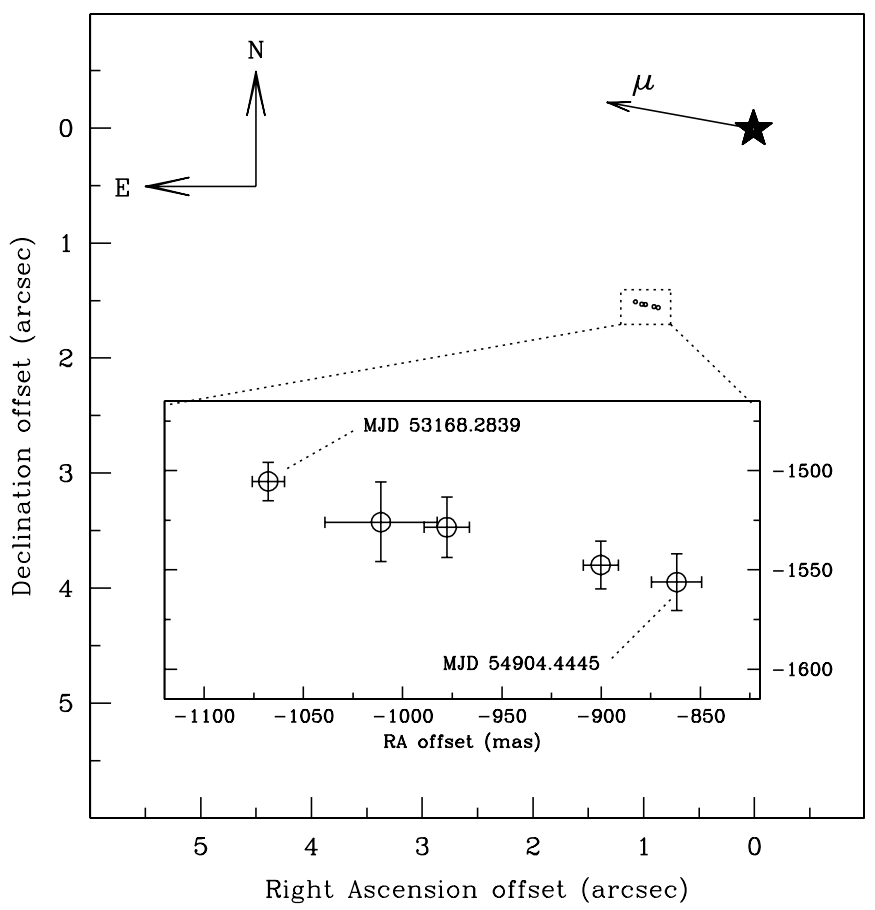

Figure 2. Offset positions of $\zeta$ Vir B relative to the host star. The position of the host star is marked with the $\star$ symbol. The inset portion of the plot shows the positions of the stellar companion over the 4.75 years of observations presented in this paper. The error bars incorporate the uncertainties in the radial separation and the position angle. The vector labeled " $\mu$ " at the upper right shows the magnitude and direction of the proper motion of the $\zeta$ Vir system over the

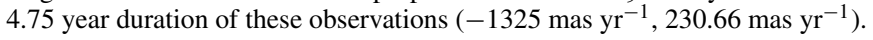

to measure the position of both the primary and the companion. $\zeta$ Vir A, listed as a high-proper-motion star, has a proper motion

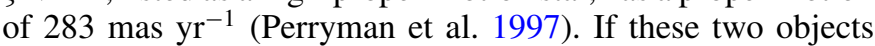
were not associated with each other, we could expect a $\sim 1^{\prime \prime} .35$ change in separation over the 4.75 year course of observations. Instead, we report a $\sim 200$ mas southwesterly change in the position of the companion (see Figure 2) relative to the host star. Since the relative separation between the host star and the companion is far less than the $\sim 1^{\prime \prime} .35$ change in separation if the two were not mutually bound, we are confident that these two objects share common proper motion. Moreover, this westerly change reflects the orbital motion of $\zeta$ Vir B over the 4.75 year observing baseline.

\subsection{Photometry}

Aperture photometry of the companion was performed on images from the Lyot Project in which $\zeta$ Vir was occulted behind our coronagraphic mask. The flux was summed in apertures of radii of 270 mas, $340 \mathrm{mas}$, and 300 mas, for the $J-, H$-, and $K$-band images, respectively, followed by sky subtraction. Zero points for the $J, H$, and $K$ data were derived by performing large (760 mas) aperture photometry on unocculted, unsaturated 


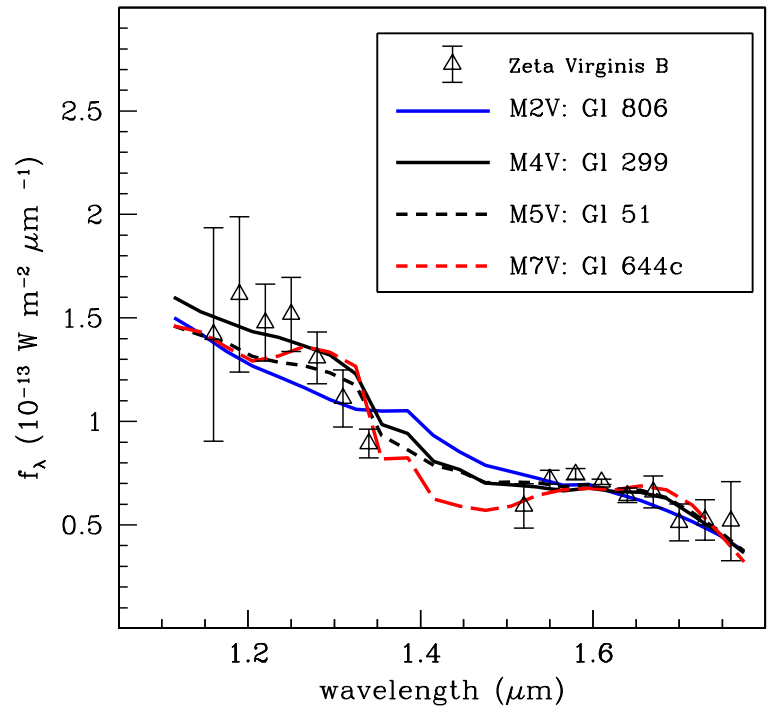

Figure 3. $J$ - and $H$-band spectra of $\zeta$ Vir B obtained with our IFS and coronagraph at Palomar (Hinkley et al. 2008). Also shown are template spectra for M2V through M7V stars taken from the IRTF spectral library (Cushing et al. 2005; Rayner et al. 2009). The water band data points between $\sim 1.35$ and $\sim 1.5 \mu \mathrm{m}$ have been excluded due to the variation of this band between observations of $\zeta$ Vir and our calibrator star.

Table 3

Photometry for $\zeta$ Virginis B

\begin{tabular}{lcc}
\hline \hline Band & Apparent Magnitude & Absolute Magnitude \\
\hline$J$ & $10.75 \pm 0.06$ & $8.99 \pm 0.06$ \\
$H$ & $10.17 \pm 0.14$ & $8.41 \pm 0.14$ \\
$K$ & $9.90 \pm 0.17$ & $8.14 \pm 0.17$ \\
\hline
\end{tabular}

images of $\zeta$ Vir A taken immediately prior to the occulted observations. We calculate $J$-, $H$-, and $K$-band photometric zero points of $20.990 \pm 0.017,20.639 \pm 0.078$, and $19.932 \pm 0.069$, respectively. Assuming a distance of $22.45 \mathrm{pc}$ (Perryman et al. 1997), Table 3 shows our calculated absolute $J-, H$-, and $K$-band magnitudes of $8.99 \pm 0.06,8.41 \pm 0.14$, and $8.14 \pm$ 0.17 , respectively, for $\zeta$ Vir B.

\subsection{Spectroscopy}

Integrating an IFS into more conventional high-contrast imaging techniques can provide significantly more information on objects in close vicinity to their host star (Sparks \& Ford 2002; Berton et al. 2006; McElwain et al. 2007; Antichi et al. 2009). Normally, when spectra are unavailable, parameters such as mass, spectral type, and age must be derived by combining broadband photometry with model predictions. Such models can be problematic at very young ages (Stassun et al. 2006; Allers et al. 2007).

Observations with our IFS at Palomar Observatory (Hinkley et al. 2008) allowed us to obtain the spectrum of $\zeta$ Vir B shown in Figure 3. Each point in the spectrum of $\zeta$ Vir B was calculated by performing aperture photometry on each image in a data cube. Examples of three such images taken from a data cube are shown in Figure 4. The photometry was obtained using a circular aperture such that the second Airy ring of the PSF was enclosed at each wavelength in the data cube. A median background sky value was calculated in a 40 mas wide annulus, just outside the photometric aperture, and subtracted from the target counts. Each flux value for $\zeta$ Vir B shown in Figure 3 is a median of five data points taken from five data cubes of $\zeta$ Vir B, and the error bars show the $1 \sigma$ spread of these five values. The spectrum was calibrated using a reference star (HIP56809, G0V, $V=6.44$ ) by comparing the measured counts of the reference star with a template G0V star (HD109358, G0V, $V=4.26$ ) taken from the IRTF spectral library (Cushing et al. 2005; Rayner et al. 2009) to derive a spectrograph response function.

The spectrum shown in Figure 3 has had this response correction applied to it. We have excluded the data points in the vicinity of the water absorption band between $\sim 1.35 \mu \mathrm{m}$ and $\sim 1.5 \mu \mathrm{m}$, since the degree of water absorption present in the calibrator star was sufficiently different from that present in the $\zeta$ Vir observations. Also shown in the figure are template spectra for an M2V through M7V star taken from the IRTF spectral library. The extracted spectrum for $\zeta$ Vir B is most consistent with the M4V-M7V spectral types.

\section{ANALYSIS}

\subsection{Mass and Age of $\zeta$ Vir A}

Although Hoffleit \& Jaschek (1982) list $\zeta$ Vir as a possible member of the Hyades moving group, several lines of evidence suggest simply assigning the system the age of the Hyades cluster is not appropriate. Indeed, using criteria based on mass distribution and metallicity, Famaey et al. (2007) question whether most members of the Hyades Moving Group are actually evaporated members of the Hyades Open Cluster.

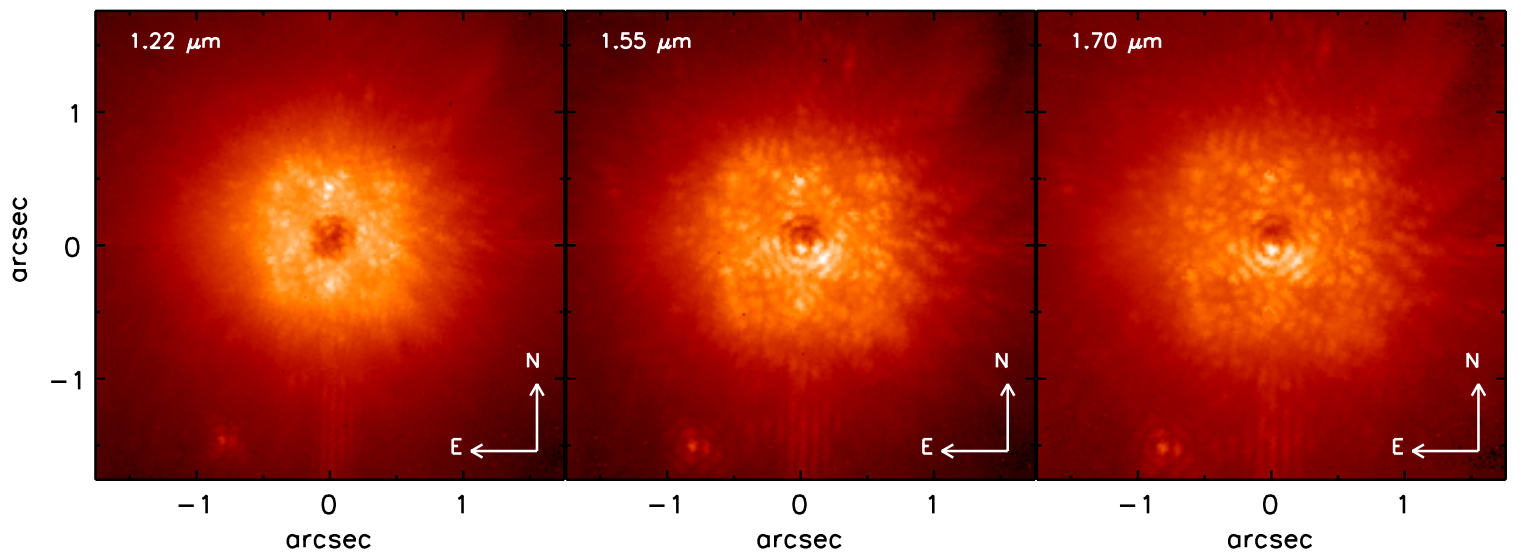

Figure 4. Three images of the $\zeta$ Vir system taken from a data cube from the Project 1640 IFS. The three images show the system at $1.22,1.55$, and $1.70 \mu \mathrm{m}$. The host star, $\zeta$ Vir A, has been coronagraphically occulted at the center of each image, and the companion, $\zeta$ Vir B, is evident at the lower left. 

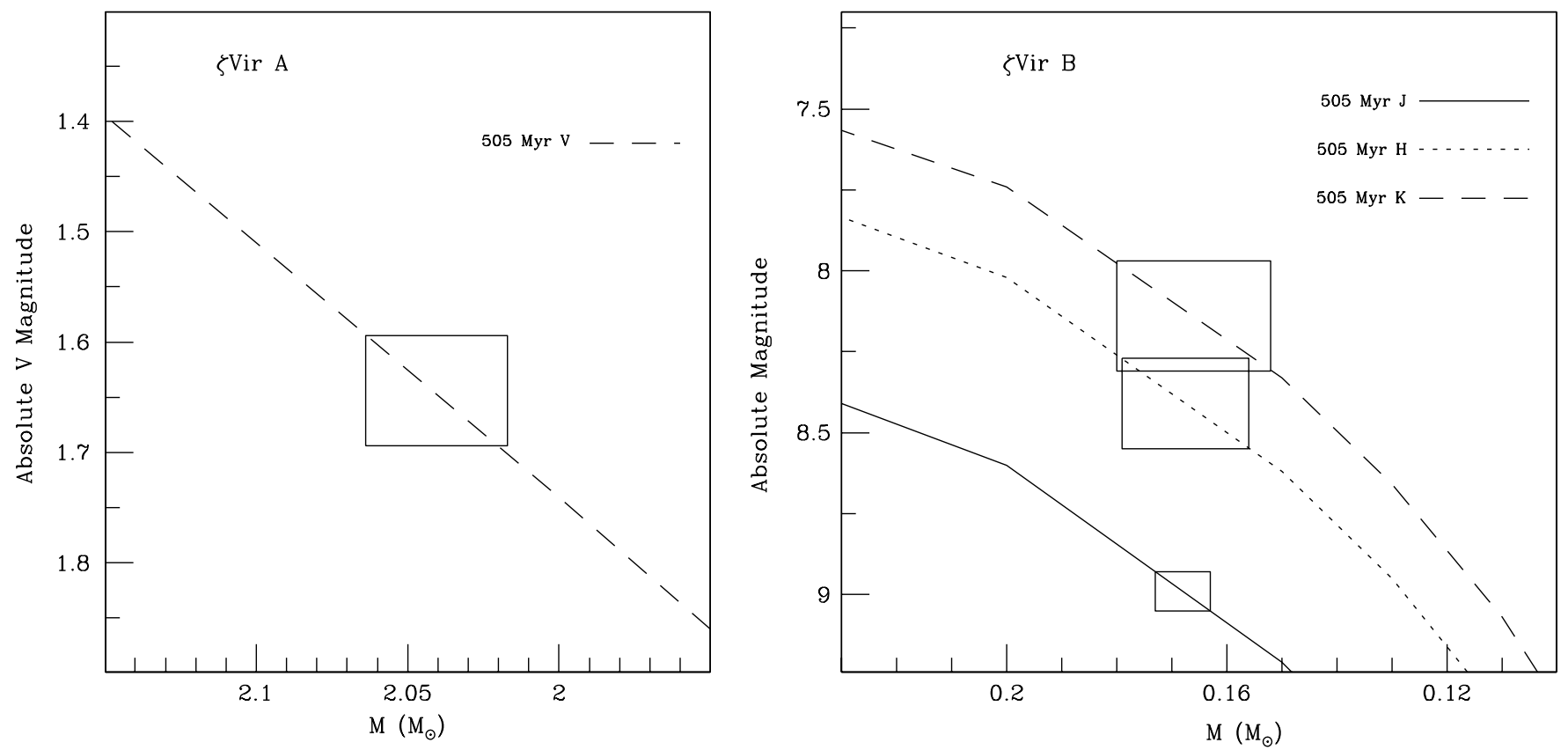

Figure 5. Left-hand panel shows the luminosity-mass relation for the host star, $\zeta$ Vir A, as calculated by Siess et al. (2000) for a $505 \mathrm{Myr}$ system. We use absolute $V$ magnitude in lieu of total luminosity. The vertical extent of the box indicates the $V$-band photometric uncertainty $\left(M_{V}=1.64 \pm 0.05\right)$ for $\zeta$ Vir A. This defines an allowable region for the mass of the A3V host star to be $2.041 \pm 0.024 M_{\odot}$. The right panel shows evolutionary models for low-mass stars taken from Baraffe et al. (2003) for the $J, H$, and $K$ bands. As with the case for $\zeta$ Vir A, the vertical extent of each box indicates the photometric uncertainty at each band. These three bandpass values give an overall value of $0.168_{-0.016}^{+0.012} M_{\odot}$ for $\zeta$ Vir B.

Table 4

$J_{-}, H$-, and $K$-band Mass Determinations for $\zeta$ Vir B

\begin{tabular}{lccccc}
\hline \hline \multicolumn{1}{c}{ Model } & Method & $J$ Band & $H$ Band & $K$ Band & Median $\pm 1 \sigma$ \\
\hline Henry \& McCarthy (1993) & Mass-Luminosity (Empirical) & $0.136 M_{\odot}$ & $0.152 M_{\odot}$ & $0.152 M_{\odot}$ & $0.152 \pm 0.009 M_{\odot}$ \\
Baraffe et al. (2003) (505 Myr) & Evolutionary (Theoretical) & $0.168 M_{\odot}$ & $0.169 M_{\odot}$ & $0.167 M_{\odot}$ & $0.168 \pm 0.001 M_{\odot}$ \\
Delfosse et al. (2000) & Mass-Luminosity (Empirical) & $0.170 M_{\odot}$ & $0.166 M_{\odot}$ & $0.163 M_{\odot}$ & $0.166 \pm 0.004 M_{\odot}$ \\
\hline
\end{tabular}

The mean metallicity of the Hyades cluster has been well established with an $[\mathrm{Fe} / \mathrm{H}]$ value of $0.14 \pm 0.05$ (Cayrel de Strobel et al. 1997), and later refined to $0.144 \pm 0.013$ (Grenon $2000)$. However, the mean metallicity value of $[\mathrm{Fe} / \mathrm{H}] \simeq-0.02$ (Gray et al. 2003) for the $\zeta$ Vir system is significantly different than the above values, ruling out the possibility that this star is a member of the Hyades cluster. For this work, we have decided to adopt the age of 505 Myr given by Rieke et al. (2005). In the left panel of Figure 5, we show the luminosity-mass parameter space showing model tracks calculated by Siess et al. (2000) for a $505 \mathrm{Myr}$ system with solar metallicity. The vertical extent of the box indicates the absolute $V$-band photometric uncertainty. This uncertainty in the luminosity $\left(M_{V}=1.64 \pm 0.05\right)$ defines an allowable mass region for $\zeta$ Vir A of $2.041 \pm 0.024 M_{\odot}$.

\subsection{Mass and Age of $\zeta$ Vir B}

We use the models of Baraffe et al. (2003) to derive a mass for $\zeta$ Vir B assuming the age of 505 Myr. In Figure 5 (right panel), we show plots of the $J-, H$-, and $K$-band absolute magnitudes for a range of companion masses calculated from these models. As with the case of $\zeta$ Vir A, the uncertainty in the photometry of this object has very little effect on the derived mass of the companion. Together these values give an overall derived mass of $0.168_{-0.016}^{+0.012} M_{\odot}$. We take this value as the final derived mass for $\zeta$ Vir B.

To check the validity of this model-based mass estimate, we compare this value with empirically derived mass-luminosity relations given in Henry \& McCarthy (1993) and Delfosse et al. (2000). Using the $J, H$, and $K$ magnitudes, these two works predict values of $0.152 \pm 0.009 M_{\odot}$ and $0.166 \pm 0.004 M_{\odot}$ (see Table 4), consistent with a mid-M spectral type for a mainsequence star at these ages (Reid et al. 1995; Hawley et al. 1996), and consistent with the spectral determination derived previously. The Henry \& McCarthy (1993) and Delfosse et al. (2000) mass values are slightly lower than the model-based $0.168 M_{\odot}$ value given by the Baraffe et al. (2003) models, but are still consistent with a mid-M spectral type for $\zeta$ Vir B. Using the range of primary star masses derived above gives this system a mass ratio of $q=0.082_{-0.008}^{+0.007}$.

\subsection{Orbital Analysis}

Given the relatively short span (4.75 years) of the observations of $\zeta$ Vir B (see Figure 2), fitting an orbital model to the data is premature. However, we may borrow an analysis used by Golimowski et al. (1998) to constrain the eccentricity, $e$, and semimajor axis, $a$, of $\zeta$ Vir B using our astrometry in the twodimensional plane of the sky, combined with Kepler's Laws. We refer the reader to Golimowski et al. (1998) for a full explanation. Over our 4.75 year time baseline, we calculate a velocity of $-1.139 \mathrm{AU} \mathrm{yr}^{-1}$ in the westward direction, and

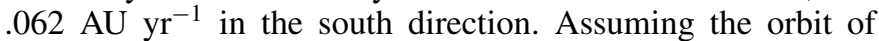
$\zeta$ Vir B is bounded, and assuming a range of line-of-sight positions and velocities for $\zeta$ Vir B, we are able to constrain $a$ and $e$, and we show the loci of possible values for these 


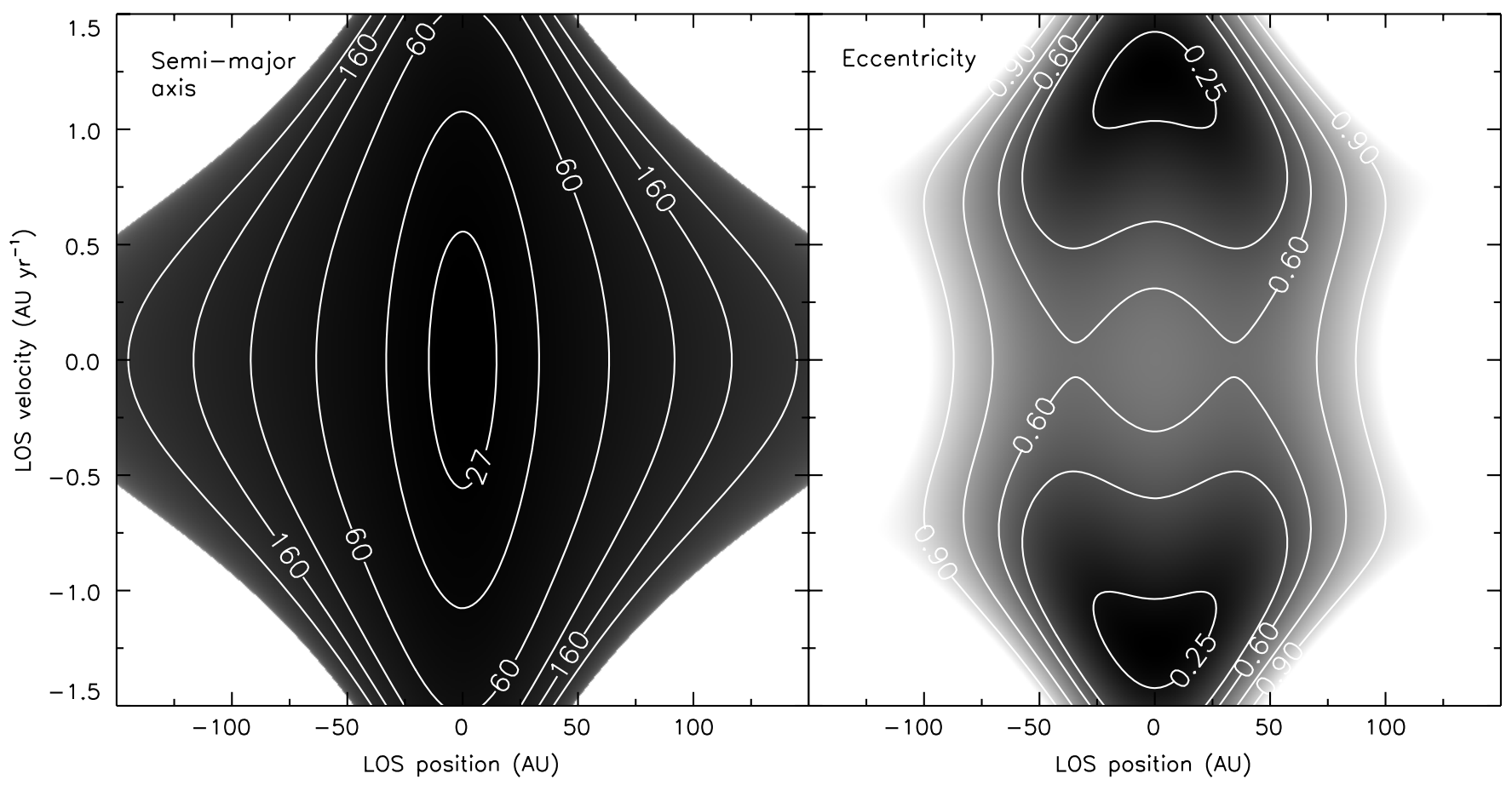

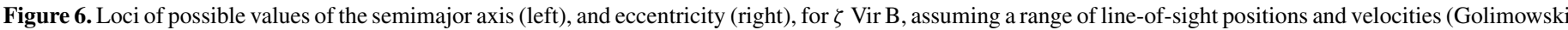

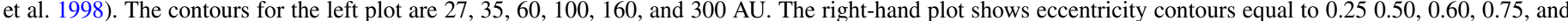
0.90 . This constrains the semimajor axis to be $\gtrsim 24.9 \mathrm{AU}$ (and hence the period to be $\gtrsim 124 \mathrm{yr}$ ), and eccentricity $\gtrsim 0.16$.

parameters in Figure 6. The ordinate and abscissa values show the assumed values of the line-of-sight velocity, and position, respectively. The values shown in Figure 6 indicate $a \gtrsim 24.9 \mathrm{AU}$ and $e \gtrsim 0.16$. From the semimajor axis constraint, we can constrain the period $P=\left(4 \pi^{2} a^{3} / \mu\right)^{1 / 2}$ to be $\gtrsim 124 \mathrm{yr}$, where $\mu=G\left(m_{1}+m_{2}\right)$. In this analysis, we have assumed a mass of $m_{1}=2.04 M_{\odot}$ (see Figure 5) and $m_{2}=0.168 M_{\odot}$ for $\zeta$ Vir A and $\zeta$ Vir B, respectively.

\section{DISCUSSION}

Assuming a mass of $2.041 \pm 0.024 M_{\odot}$ for $\zeta$ Vir A gives this newly discovered binary system a mass ratio of $q=0.082_{-0.008}^{+0.007}$. Although numerous low-mass companions have been detected around $\sim 1 M_{\odot}$ stars, this system is of particular interest given that it orbits a primary star of $\sim 2 M_{\odot}$. The $q$ distributions for primaries in the mid- and low-mass stellar regimes have been well studied, but comprehensive binary statistics for A stars are incompletely surveyed. The mass ratio distribution for the mid and lower mass ranges show fairly clear trends with mass. Namely, Burgasser et al. (2007) discuss that very low mass binaries have mass ratios that are skewed toward unity. Studies using a complete sample of stars between 0.6 and $0.85 M_{\odot}$ (Mazeh et al. 2003), as well as M-dwarf surveys (Fischer \& Marcy 1992; Reid \& Gizis 1997), find a significantly flat distribution of mass ratios. In the same vein, Kraus et al. (2008) used a sample of 82 young stars of GKM type in the Upper Sco star-forming region and found a distribution of mass ratios not significantly different from a constant distribution, i.e., not significantly biased toward having low-mass companions. Toward higher masses, Duquennoy \& Mayor (1991) find that F- and G-type stars have a broad range of mass ratios, but with a slight increase toward small secondary masses. Finally, at the higher mass end, Shatsky \& Tokovinin (2002) and Kouwenhoven et al. (2005) have performed a survey of A and B stars in the Sco OB2 association, finding a high rate of binarity.

Similarly, many studies show a positive correlation between the distribution of binary separations and the mass of the system. The Duquennoy \& Mayor (1991) sample of solar-mass stars show a mean separation of $\sim 30$ AU. At lower masses, the Fischer \& Marcy (1992) survey (largely M dwarfs within 8 pc) and Reid \& Gizis (1997) surveys (M dwarfs within 20 pc) find mean separations between 4 and 30 AU. Continuing toward lower masses, low-mass $\mathrm{M}$ dwarfs and brown dwarfs as discussed in Burgasser et al. (2007), have notably smaller mean separations $(\sim 4 \mathrm{AU})$, with maximum separations $\sim 20 \mathrm{AU}$.

Kouwenhoven et al. (2005) and Shatsky \& Tokovinin (2002) have undertaken the first steps toward a comprehensive study of the multiplicity of A stars. Our work aims to aid in that effort. The value of the current study lies in the ability to obtain a spectrum which determines the spectral type, a tight constraint on the secondary mass, and a constrained orbit.

Our finding may also be useful for studies of the anomalous $\mathrm{X}$-ray emission from A stars. Unseen late-type companions to A stars have been hypothesized to be the source of their anomalous $\mathrm{X}$-ray emission. Indeed, the presence of the M-dwarf companion in this system can easily account for the X-ray flux detected by ROSAT. For a $0.168 M_{\odot}$ star at $505 \mathrm{Myr}$, the Baraffe et al. (2003) evolutionary tracks predict a luminosity of $L_{\text {bol }} \simeq 2 \times$ $10^{31} \mathrm{erg} \mathrm{s}^{-1}$. And assuming that the X-ray luminosity $L_{x}=$ $1.07 \times 10^{28} \mathrm{erg} \mathrm{s}^{-1}$ noted by Huensch et al. (1998) is due completely to the companion, this predicts a $\log \left(L_{x} / L_{\text {bol }}\right)$ value of -3.3 , quite typical for a young mid-M dwarf (see Fleming et al. 1993, especially their Figure 3). Recently, Zimmerman et al. (2010) have reported the presence of a mid-M dwarf bound to the star Alcor. More complete highcontrast surveys for companions surrounding an ensemble of A stars will allow researchers to begin to address the issue 
of the anomalous X-ray emission in a statistically robust manner.

An abundance of M-dwarf companions in configurations like this also may lend support to models of binary formation based on fragmentation of a massive circumstellar disk. As Kratter et al. (2010) point out, massive stars with their presumably massive circumstellar disks and correspondingly high-mass infall rates provide an environment conducive for the formation of disk instabilities and fragmentation. Indeed, such a mechanism is more likely for disks surrounding stars more massive than 1-2 $M_{\odot}$ (Krumholz et al. 2007; Kratter et al. 2008). If indeed this fragmentation is a prominent mechanism for the formation of binary companions (Stamatellos \& Whitworth 2009), the abundance of low-mass companions (brown dwarfs and M-dwarfs) should be more frequent around more massive stars.

\section{SUMMARY}

We report the discovery of a low-mass, M4V-M7V stellar companion to the star $\zeta$ Vir. This object clearly shares common proper motion with its host star, and we derive a mass of $0.168_{-0.016}^{+0.012} M_{\odot}$, corresponding to a mass ratio $q=0.082_{-0.008}^{+0.007}$. Our broadband photometry and spectroscopy are consistent with a mid-M spectral type. Although numerous low-mass companions have been identified around $\sim 1 M_{\odot}$ systems, this object is significant given its membership in a $\sim 2 M_{\odot}$ system. Characterization of more systems like this is important for identifying the anomalous source of X-rays from A stars as well as constraining possible modes of formation of stellar companions through the fragmentation of massive circumstellar disks.

We thank the anonymous referee for his or her comments. This work was performed in part under contract with the California Institute of Technology (Caltech) funded by NASA through the Sagan Fellowship Program. The Lyot Project is based upon work supported by the National Science Foundation under grants AST-0804417, 0334916, 0215793, and 0520822, as well as grant NNG05GJ86G from the National Aeronautics and Space Administration under the Terrestrial Planet Finder Foundation Science Program. A portion of the research in this paper was carried out at the Jet Propulsion Laboratory, California Institute of Technology, under a contract with the National Aeronautics and Space Administration and was funded by internal Research and Technology Development funds. The Lyot Project gratefully acknowledges the support of the US Air Force and NSF in creating the special Advanced Technologies and Instrumentation opportunity that provides access to the AEOS telescope. Eighty percent of the funds for that program are provided by the US Air Force. This work is based on observations made at the Maui Space Surveillance System, operated by Detachment 15 of the U.S. Air Force Research Laboratory Directed Energy Directorate. This work has been partially supported by the NSF Science and Technology Center for Adaptive Optics, managed by the University of California at Santa Cruz under cooperative agreement AST 98-76783. The Lyot Project is also grateful to the Cordelia Corporation, Hilary and Ethel Lipsitz, the Vincent Astor Fund, Judy Vale, and an anonymous donor, who initiated the project.

\section{REFERENCES}

Abt, H. A., \& Biggs, E. S. 1972, Bibliography of Stellar Radial Velocities (New York: Latham Process Corp.)

Akeson, R. L., et al. 2009, ApJ, 691, 1896
Allers, K. N., et al. 2007, ApJ, 657, 511

Antichi, J., et al. 2009, ApJ, 695, 1042

Baraffe, I., Chabrier, G., Barman, T. S., Allard, F., \& Hauschildt, P. H. 2003, A\&A, 402, 701

Berton, A., Gratton, R. G., Feldt, M., Henning, T., Desidera, S., Turatto, M., Schmid, H. M., \& Waters, R. 2006, PASP, 118, 1144

Burgasser, A. J., Reid, I. N., Siegler, N., Close, L., Allen, P., Lowrance, P., \& Gizis, J. 2007, in Protostars and Planets V, ed. B. Reipurth, D. Jewitt, \& K Keil (Tucson, AZ: Univ. of Arizona Press), 427

Burkert, A. \& Bodenheimer, P. 1996, MNRAS, 280, 1190

Cayrel de Strobel, G., Crifo, F., \& Lebreton, Y. 1997, in ESA Special Publication, Vol. 402, Hipparcos-Venice '97 (Noordwijk, Netherlands: ESA), 687

Cushing, M. C., Rayner, J. T., \& Vacca, W. D. 2005, ApJ, 623, 1115

Dekany, R. G., Brack, G., Palmer, D., Oppenheimer, B. R., Hayward, T. L., \& Brandl, B. 1998, Proc. SPIE, 3353, 56

Delfosse, X., Forveille, T., Ségransan, D., Beuzit, J.-L., Udry, S., Perrier, C., \& Mayor, M. 2000, A\&A, 364, 217

Digby, A. P., et al. 2006, ApJ, 650, 484

Duquennoy, A., \& Mayor, M. 1991, A\&A, 248, 485

Famaey, B., Pont, F., Luri, X., Udry, S., Mayor, M., \& Jorissen, A. 2007, A\&A, 461, 957

Fischer, D. A., \& Marcy, G. W. 1992, ApJ, 396, 178

Fleming, T. A., Giampapa, M. S., Schmitt, J. H. M. M., \& Bookbinder, J. A. 1993, ApJ, 410, 387

Golimowski, D. A., Burrows, C. J., Kulkarni, S. R., Oppenheimer, B. R., \& Brukardt, R. A. 1998, AJ, 115, 2579

Golimowski, D. A., et al. 2006, AJ, 131, 3109

Gray, R. O., Corbally, C. J., Garrison, R. F., McFadden, M. T., \& Robinson, P. E. 2003, AJ, 126, 2048

Grenon, M. 2000, IAU Joint Discussion, Vol. 13

Hartkopf, W. I., Mason, B. D., \& Worley, C. E. 2001, AJ, 122, 3472

Hawley, S. L., Gizis, J. E., \& Reid, I. N. 1996, AJ, 112, 2799

Heinze, A. N., Hinz, P. M., Kenworthy, M., Miller, D., \& Sivanandam, S. 2008, ApJ, 688, 583

Henry, T. J., \& McCarthy, D. W., Jr. 1993, AJ, 106, 773

Hinkley, S., Oppenheimer, B. R., Brenner, D., Parry, I. R., Sivaramakrishnan, A., Soummer, R., \& King, D. 2008, Proc. SPIE, 7015, 701519

Hinkley, S., et al. 2007, ApJ, 654, 633

Hinkley, S., et al. 2009, ApJ, 701, 804

Hinz, P. M., Heinze, A. N., Sivanandam, S., Miller, D. L., Kenworthy, M. A., Brusa, G., Freed, M., \& Angel, J. R. P. 2006, ApJ, 653, 1486

Hoffleit, D., \& Jaschek, C. 1982, The Bright Star Catalogue (4th ed.; New Haven, CT: Yale Univ. Observatory)

Huensch, M., Schmitt, J. H. M. M., \& Voges, W. 1998, A\&AS, 132, 155

Johnson, J. A., Butler, R. P., Marcy, G. W., Fischer, D. A., Vogt, S. S., Wright, J. T., \& Peek, K. M. G. 2007, ApJ, 670, 833

Kalas, P., Graham, J. R., \& Clampin, M. 2005, Nature, 435, 1067

Kalas, P., et al. 2008, Science, 322, 1345

Kilkenny, D., O’Donoghue, D., Koen, C., Lynas-Gray, A. E., \& van Wyk, F. 1998, MNRAS, 296, 329

Kouwenhoven, M. B. N., Brown, A. G. A., Zinnecker, H., Kaper, L., \& Portegies Zwart, S. F. 2005, A\&A, 430, 137

Kratter, K. M., Matzner, C. D., \& Krumholz, M. R. 2008, ApJ, 681, 375

Kratter, K. M., Murray-Clay, R. A., \& Youdin, A. N. 2010, ApJ, 710, 1375

Kraus, A. L., Ireland, M. J., Martinache, F., \& Lloyd, J. P. 2008, ApJ, 679, 762

Krumholz, M. R., Klein, R. I., \& McKee, C. F. 2007, ApJ, 656, 959

Lagrange, A.-M., Desort, M., Galland, F., Udry, S., \& Mayor, M. 2009, A\&A, 495,335

Lebreton, Y., Fernandes, J., \& Lejeune, T. 2001, A\&A, 374, 540

Lyot, B. 1939, MNRAS, 99, 580

Marois, C., Macintosh, B., Barman, T., Zuckerman, B., Song, I., Patience, J., Lafrenière, D., \& Doyon, R. 2008, Science, 322, 1348

Mazeh, T., Simon, M., Prato, L., Markus, B., \& Zucker, S. 2003, ApJ, 599, 1344 McAlister, H. A., Mason, B. D., Hartkopf, W. I., \& Shara, M. M. 1993, AJ, 106, 1639

McDonald, J. M., \& Clarke, C. J. 1993, MNRAS, 262, 800

McElwain, M. W., et al. 2007, ApJ, 656, 505

Oppenheimer, B. R., \& Hinkley, S. 2009, ARA\&A, 47, 253

Oppenheimer, B. R., et al. 2004, Proc. SPIE, 5490, 433

Oppenheimer, B. R., et al. 2008, ApJ, 679, 1574

Patience, J., Macintosh, B. A., \& Max, C. E. 2001, Proc. SPIE, 4490, 178

Perrin, M. D., Graham, J. R., Trumpis, M., Kuhn, J. R., Whitman, K., Coulter, R., Lloyd, J. P., \& Roberts, L. C. 2003, in 2003 AMOS Technical Conf., ed. P. W. Kervin \& J. L. Africano (Kihei, HI: Maui Economic Development Board)

Perryman, M. A. C., et al. 1997, A\&A, 323, L49

Perryman, M. A. C., et al. 1998, A\&A, 331, 81 
Rayner, J. T., Cushing, M. C., \& Vacca, W. D. 2009, ApJS, 185, 289

Reid, I. N., \& Gizis, J. E. 1997, AJ, 113, 2246

Reid, I. N., Hawley, S. L., \& Gizis, J. E. 1995, AJ, 110, 1838

Rieke, G. H., et al. 2005, ApJ, 620, 1010

Roberts, L. C., \& Neyman, C. R. 2002, PASP, 114, 1260

Schmitt, J. H. M. M., Golub, L., Harnden, F. R., Jr., C. W., Maxson, Rosner, R., \& Vaiana, G. S. 1985, ApJ, 290, 307

Schröder, C., \& Schmitt, J. H. M. M. 2007, A\&A, 475, 677

Shatsky, N., \& Tokovinin, A. 2002, A\&A, 382, 92

Siess, L., Dufour, E., \& Forestini, M. 2000, A\&A, 358, 593

Sivaramakrishnan, A., Koresko, C. D., Makidon, R. B., Berkefeld, T., \& Kuchner, M. J. 2001, ApJ, 552, 397
Sivaramakrishnan, A., \& Oppenheimer, B. R. 2006, ApJ, 647, 620

Sivaramakrishnan, A., et al. 2007, C. R. Phys., 8, 355

Smith, B. A., \& Terrile, R. J. 1984, Science, 226, 1421

Soummer, R. 2005, ApJ, 618, L161

Soummer, R., et al. 2006, in Astronomy with High Contrast Imaging II: Instrumental Techniques, Modeling, and Data Processing, ed. M. Carbillet, A. Ferral, \& C. Aime (Les Ulis: EDP Sciences), 199

Sparks, W. B., \& Ford, H. C. 2002, ApJ, 578, 543

Stamatellos, D., \& Whitworth, A. P. 2009, MNRAS, 392, 413

Stassun, K. G., Mathieu, R. D., \& Valenti, J. A. 2006, Nature, 440, 311

Zimmerman, N., et al. 2010, ApJ, 709, 733 